\title{
A New Indicator for Gain-Switching Control of Position Error-based Teleoperation System
}

\author{
Kamran Razi \\ Depart. of Electrical Eng. \\ Amirkabir Univ. of Tech. \\ Tehran, Iran \\ Kamranrazi@ut.ac.ir
}

\author{
Reza Monfaredi \\ Depart. of Mechanical Eng. \\ Amirkabir Univ. of Tech. \\ Tehran, Iran \\ Rezamonfaredi@aut.ac.ir
}

\author{
Saeed Shiri Ghydari \\ Depart. of Computer Eng. \\ Amirkabir Univ. of Tech. \\ Tehran, Iran \\ shiry@ce.aut.ac.ir
}

\author{
S. M. Rezaei \\ Depart. of Mechanical Eng. \\ Amirkabir Univ. of Tech. \\ Tehran, Iran \\ Smrezaei@cic.aut.ac.ir
}

\begin{abstract}
Some adaptive control schemes was suggested for Teleoperation systems. These schemes are based on environment impedance estimation which has abrupt changes. In this paper, estimation loop is applied to identify just gradual variations in the slave robot dynamic. Then the required data for compensating environment changes are explored through accurate slave dynamic. Experimental results for both negligible time delay and $150 \mathrm{~ms}$ time delay in the communication channel are included to show the effectiveness of the proposed controller. Also, stability analysis for the designed controller is presented.
\end{abstract}

Index Terms- Teleoperation, Transparency, gain switching controller.

\section{INTRODUCTION}

Teleoperation systems have been applied in many areas such as hazardous environments, demining, surgical operation, and etc to ensure accuracy and safety. The stabilityPerformance trade-off is the main determinant of the control design for teleoperation systems and these two factors inherently depend on the task for which the system is designed. These robotic systems have limited flexibility in adapting to work with environmental changes. Besides stability problems, in order to evaluate the performance of the system quantitatively, an appropriate performance measure must be selected. The telepresence is another common goal for designing a control system for these devices. Telepresence is a sense of the operator who is working with a teleoperation system and it is desired to be felt as if one were doing the task directly. Fig.1 shows general structure of teleoperation systems.

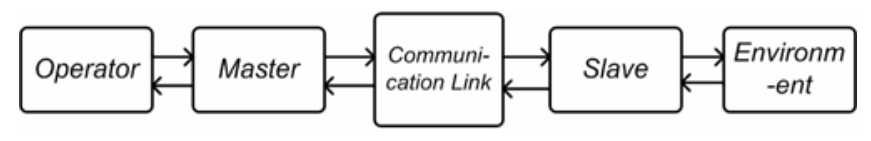

Fig. 1 Teleoperatory System General Structure

Besides telepresence and stability problems, bandwidth and response requirements must be concerned for designing effective teleoperation system [12]. In order to achieve a high degree of transparency in performing different tasks with special characteristics, several works have been done.
Lawrence [4] used four-channel architecture for system and defined the mathematical definition for ideal transparency. He proposed a time invariant controller using force, position, and velocity signals at both side. Moreover, it needs accurate master and slave dynamic to obtain four-channel transfer functions. The resulting ideal controller of Lawrence work was equivalent to Yokokohji's [14] ideal teleoperation controller. H-Zaad et al [7] incorporated local force feedback and suggested some benefits in the terms of stability and transparency. Rather than ideal transparency, M.c.Cavusoglu et al [5] introduced a new measure of fidelity in teleoperation, which quantifies the teleoperation system's ability to transmit changes in the compliance of the environment. They proposed that it is important to use a task based performance objective rather than seeking for generic ideal teleoperation response.

Hannaford [8] suggested a 2-port hybrid network description model for teleoperation system, which is widely used. Fig.2 shows this network. Impedance which is transmitted to the operator could be calculated by using this structure and hybrid model's parameters. The environment impedance transmitted through the teleoperator is calculated as in (1). It should be noted that the impedances are defined as Force/Position not Force/velocity. Typically, the environment and the human impedance have nonlinear dynamics; however they are often modelled by Linear Time Invariant differential equations to enable the application of linear theory for analysis and synthesis [13].

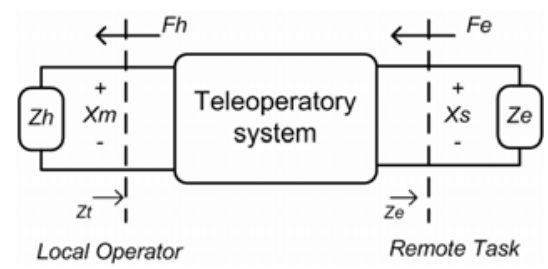

Fig. 2 Two Port Hybrid Network

$$
\begin{aligned}
& {\left[\begin{array}{l}
F_{h}(s) \\
V_{h}(s)
\end{array}\right]=\left[\begin{array}{ll}
H_{11}(s) & H_{12}(s) \\
H_{21}(s) & H_{22}(s)
\end{array}\right] \times\left[\begin{array}{c}
V_{e}(s) \\
-F_{e}(s)
\end{array}\right]} \\
& Z_{t}=\left(H_{11}-H_{12} Z_{e}\right)\left(H_{21}-H_{22} Z_{e}\right)^{-1}
\end{aligned}
$$


Niemeyer et al. [11] have suggested a hybrid control method by employing an adaptive scheme for slave free motion and stiffness control. Their control scheme enables the system to achieve stable contact with any passive environment. Hannaford [8] analysed stability in terms of definition of time domain passivity [6], and designed a passivity controller to achieve stable condition.

In order to eliminate force measuring several adaptive control architectures have been proposed. H-zaad et al. [2] proposed an adaptive method that does not require force sensing. Instead it obtains the contact information through estimation of environment impedance. In order to show the effectiveness of their method, they presented a numerical solution. $\mathrm{Ni}$ and Wang designed gain-switching control scheme for position error-based teleoperation. Similar to HZaad's work their switching criterion is based on slave side impedance estimation. Due to the fact that environmental impedance has abrupt changes in Slave side, time required estimating this impedance causes degrading effect on both stability and performance. Furthermore, in order to estimate this impedance using their method, typical mass-damperspring model is fitted to the impedance model. However there are various nonlinear impedances which might be manipulated. This paper is motivated by these two points.

To solve these two problems, system identification loop is applied only to identify gradual changes in slave's dynamic rather than estimating environment impedance. Data required to compensate environment impedance variations is explored through environment force observation using accurate slave dynamic model. By applying this signal, both tracking desired trajectory in slave end effecter and tracking environmental force in master side could be achieved. Further explanation, equating first, master and slave position and second, the environmental force and force exerted to human leads to ideal transparency in teleoperatory systems. This adaptive method is formulated for the special case which slave robot moves in a free workspace and contacts a hard environment.

The paper is organized as follows: In section 2 we formulated slave manipulator dynamic identification. Section 3 based on gain switching controller proposed in [1] presents controller design and a new contact indicator. In section 4, experimental results for both negligible channel time delay and in presence of time delay are included, followed by contact transition stability analyses in section 5. Finally section 6 draws conclusion.

\section{ENVIRONMENT FORCE OBSERVATION}

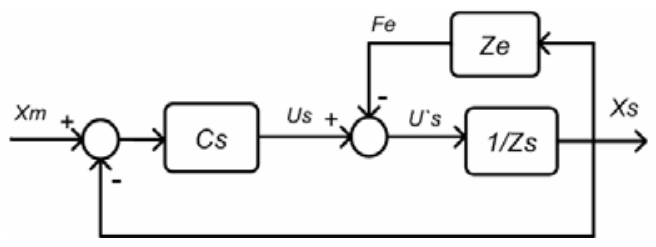

Fig.3 Slave Robot Block Diagram
In the absence of friction and other disturbances, the dynamic of a rigid one degree of freedom manipulator can be written as

$$
m_{s} x_{s}+b_{s} x_{s}=f-f_{e}
$$

where $f e$ is the force exerted on the environment and $f$ is the applied force. Fig.3 shows the block diagram of the slave system:

The summing junction equation is as follows:

$$
U_{s}-F_{e}=Z_{s} X_{s}
$$

where $Z s$ is slave dynamic. $f e$ can be observed through this equation. Inaccurate slave manipulator parameters arises a problem in estimating fe through using slave inverse dynamic model. Thus applying an identification method in order to estimate gradual changes in slave dynamics seems inevitable. Here, we drive the continuous model for identification process. Consider the transfer function from $U$ `s to Vs:

$$
\begin{gathered}
U_{s}^{\prime}=V_{s} \times\left(m_{s} s+b_{s}\right), \quad U_{s}^{\prime}=U_{s}-F_{e} \\
\Rightarrow s V_{s}=\left[\begin{array}{ll}
b_{s} / m_{s} & 1 / m_{s}
\end{array}\right] \times\left[\begin{array}{c}
-V_{s} \\
U_{s}^{\prime}
\end{array}\right]
\end{gathered}
$$

Since it is undesirable to take derivative, a stable filtering transfer function is applied.

Where

$$
s V_{e f}(t)=\theta^{T} \times \phi(t)
$$

$$
\begin{gathered}
\phi(t)=\left[\begin{array}{ll}
-V_{s f} & U_{s f}
\end{array}\right]^{T}, \quad \theta^{T}=\left[\begin{array}{ll}
b_{s} / m_{s} & 1 / m_{s}
\end{array}\right] \\
V_{s f}(t)=H_{f} \cdot V_{s}(t) \quad U_{s f}^{\prime}(t)=H_{f} \cdot U_{s}^{\prime}(t)
\end{gathered}
$$

and $H f$ is a stable transfer function with a pole excess of one.

Using RLS with exponential forgetting factor the parameter estimation is obtained using following update laws:

$$
\begin{aligned}
& \frac{d \hat{\theta}(t)}{d t}=P(t) \phi(t)\left(s V_{s f}(t)-\hat{\theta}^{T} \times \phi(t)\right) \\
& \frac{d P(t)}{d t}=\alpha P(t)-P(t) \phi(t) \phi^{T}(t) P(t)
\end{aligned}
$$

If control signal i.e. Us is persistently exciting correct parameter estimation is obtained.

To realize ZsVs, it is necessary to calculate $\dot{v}_{s}(t)$ as the second order differentiation of slave end effecter position. This leads to enter additional noise to the system and subsequently causes degrading effects on the system performance. To avoid this, the filtering method in [2] can be applied. Such a filtering was used in [1] experimentally.

$$
Z_{s} V_{s}=\omega(s) b_{s} V_{s}+\alpha\left(V_{s}-\omega(s) . V_{s}\right)
$$


There is just one final point that needs to be settled. $u_{s}=u_{s}-f_{e}$ is not available since no force measuring is applied. Hence identifying gradual changes in system's parameters is done just at the free motion time where $u_{s}=u_{s}$.

\section{CONTROLLER DESIGN}

The problem of oscillating contact, when a robot manipulator contacts with a rigid environment, occurs in position error-based teleoperation system [9]. Sufficient stability condition for this class of controller to remain stable while contact with the environment after the finite number of switching is suggested in [10]. Ni and Wang designed gainswitching control system for position error-based teleoperation. They reported no chattering in experiments with this controller, but stability discussion was left to future. Fig.4 shows the block diagram of the proposed controller. They analysed complete transparency in two extreme cases-free motion and hard contact, and suggested low master gains and high slave gains as a condition for free motion transparency and the reverse case as a condition for hard contact. They showed analytically that choosing this control policy leads to transparent teleoperation (this can be obtained easily by deriving hybrid parameters in Eq.1 for system in Fig.4). As an indicator, a linear combination of the estimated slave side impedance parameters $(\delta)$ was used for gain switching. In the absence of communication channel delay, the reported required time for $\delta$ to reach the predetermined value for gain switching was $100 \mathrm{~ms}$. This delay has a degrading effect on both transparency and stability, which is caused by position error between master and slave sides, and when combined with communication channel delay increases oscillation in

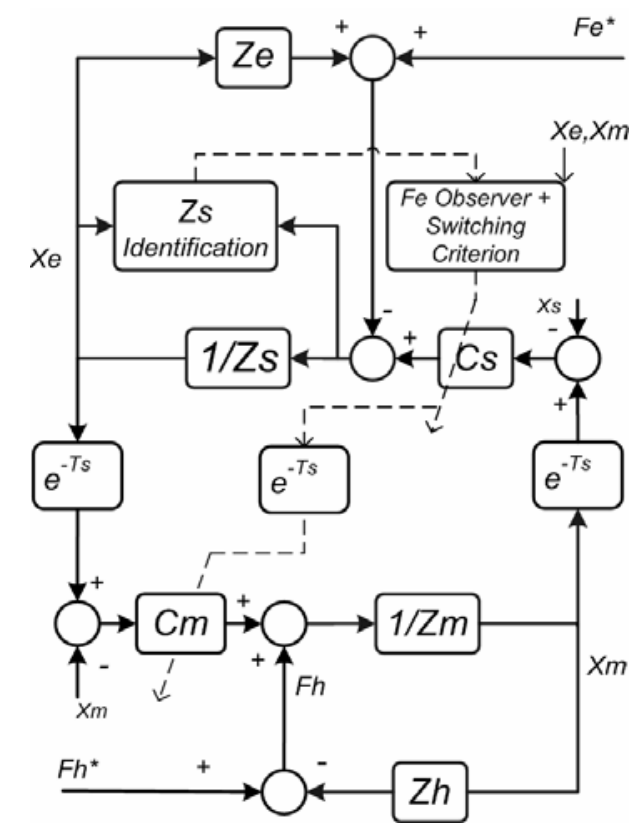

Fig.4 Gain-switching Control System Block Diagram both sides.

As a solution to this problem, another contact indicator is used as shown below.

$\left\{\begin{array}{l}\operatorname{IF}\left(F_{e}>\text { up } \_ \text {threshold }\right) \longrightarrow \text { switch_to_hard_contact_gains } \\ \operatorname{IF}\left(F_{e}<\text { down_threshold }\right) \longrightarrow \text { switch_to_free_motion_gains }\end{array}\right.$

where $F^{\prime} e$ is observed environment force. Note that the controller can be decomposed into two parts, First, the contact indicator and Second, the master and slave controllers.

The value of the up_threshold is determined by considering the saturation level of the slave motor force. Close examination of the manipulator reveals that hard contact is an inherent characteristic for every system and depends on the actuator's maximum force amplitude. If there is no friction compensation in the system, the down_threshold can be set to zero value. In our experimental setup the value of down_threshold is set to $0.1 \mathrm{~N}$ and the value of up_threshold is set to $3 \mathrm{~N}$.

\section{EXPERIMENTAL RESULTS}

Before presenting the results we briefly discuss the experimental equipment. The equipment used is a two DOF manipulator as a slave robot and a two DOF as a master robot. Each robot's controller board consists of two dc motor driver and Atmega16L Microcontroller. The master and slave controller set to be $C m=1$ and $C s=100+.5 \mathrm{~s}$ in free motion and vice versa in hard contact. Experimental setup is shown in Fig.5.

Two sets of experiments were carried out. In the first one, channel delay supposed to be negligible. The robot is touching a hard wall in position 6. In the presence of the PE signal, offline identification was applied for better parameter initialization during the experiment. Fig.6 depicts the results.

In the presence of a 150ms time delay for all three channels showed in Fig.4, the controller performs robustly as demonstrated in Fig.7, though overshoot in master position

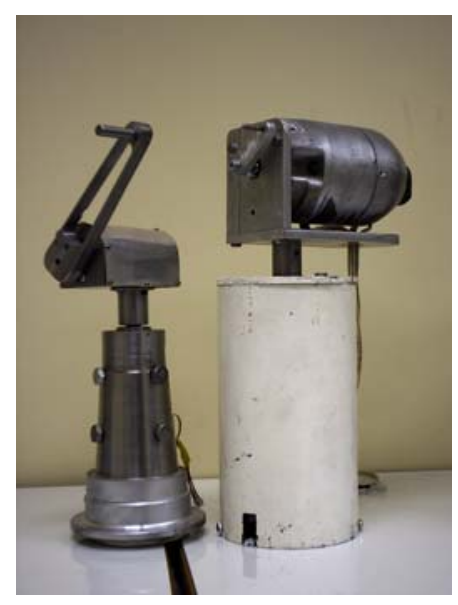

Fig.5 Experimental Setup 

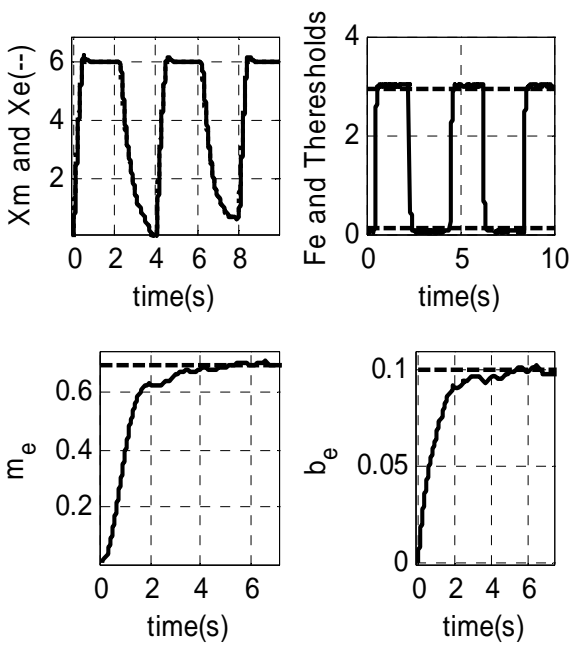

Fig.6 System response to hard contact. Position unit is 5 degrees.

signal is seen. This can be interpreted as follows: since the contact indicator leads to switching gain after a time delay, during this interval the operator moves the master arm with low gains at the master side, and thus feels free motion. After switching, the controller tries to keep the position error to zero. We mentioned earlier that the key idea of the designed controller is to omit the required time for environment impedance estimation.

\section{STABILITY DISCUSSION}

Considering Fig.4, the master dynamic is given by the linear time invariant equation of the form

$$
F_{h}^{*}+U_{m}=X_{m}\left(Z_{h}+Z_{m}\right)
$$

and the slave dynamic is given by the linear time-varying equation of the form

$$
U_{s}=X_{e}\left(Z_{s}+Z_{e} u_{\text {contact }}\right)
$$

where $u_{\text {contact }}$ equals to one where hard contact occurs and zero in free motion. Ze is stiff environment and can be modelled by a linear spring-damper system with: $b e+k e / s$.

Thus at any time instant the plant dynamics correspond to one of the two linear systems

$$
\dot{x}_{p}(t)=A_{k} x_{p}(t)+b_{k} u(t) \text { where } k=\left\{\begin{array}{l}
1(\text { free motion }) \\
2(\text { hard contact })
\end{array}\right.
$$

where

$$
\begin{aligned}
& x_{p}=\left(\begin{array}{llll}
x_{m} & \dot{x}_{m} & x_{e} & \dot{x}_{e}
\end{array}\right)^{T}, \\
& u=\left(\begin{array}{lll}
u_{m} & u_{s} & f_{h}^{*}
\end{array}\right)^{T}, \quad b_{k}=\left[\begin{array}{l}
0100 \\
0001 \\
0100
\end{array}\right]^{T}
\end{aligned}
$$
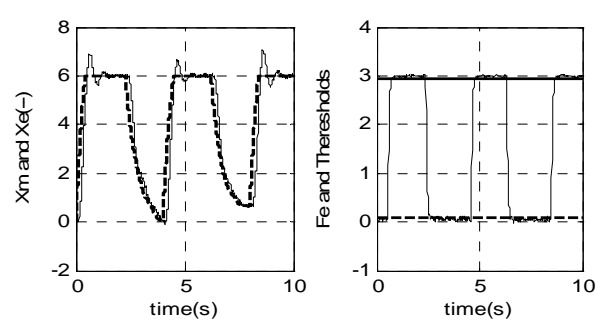

Fig.7 System response in the presence of time delay. Position unit is 5 degrees.

$$
A_{k}=\left[\begin{array}{cccc}
0 & 1 & 0 & 0 \\
-k_{h} / m_{h}+m_{m} & -b_{h}-b_{m} / m_{h}+m_{m} & 0 & 0 \\
0 & 0 & 0 & 1 \\
0 & 0 & -k_{e} \delta_{2 k}-k_{s} / m_{s}-b_{e} \delta_{2 k}-b_{s} / m_{s}
\end{array}\right]
$$

where $\delta_{i j}$ denotes the Kronecker symbol.

As mentioned in the controller design section, In order to achieve the design objectives two sets of controllers were designed for each case. In general, the dynamics of each set of controllers are given by

$$
\begin{aligned}
& \dot{x}_{k}(t)=K_{k} x_{k}(t)+L_{k} x_{p}(t) \\
& u(t)=M_{k} x_{k}(t)
\end{aligned}
$$

The state $x$ of the switched closed-loop system consists of the plant-states $x_{p}$ and the controller-states $x_{k}$

$$
x=\left(\begin{array}{lll}
x_{p}^{T} & x_{1}^{T} & x_{2}^{T}
\end{array}\right)
$$

For the switched system we then obtain

$$
\dot{x}(t)=H(t) x(t)
$$

where $H($.$) is a constant function H: \mathfrak{R} \rightarrow\left\{\begin{array}{ll}H_{1} & H_{2}\end{array}\right\}$. Then $H_{k}$ is given by

$$
H_{k}=\left[\begin{array}{ccc}
A_{k} & b_{k}^{\prime} M_{k} \delta_{k 1} & b_{k}^{\prime} M_{k} \delta_{k 2} \\
L_{1} & K_{1} & 0 \\
L_{2} & 0 & K_{2}
\end{array}\right]
$$

and $b_{k}^{\prime}$ represents the first two columns of $b_{k}$.

The closed loop system is exponentially stable if and only if the switched system formed by the matrices $H k, k=1,2$ is exponentially stable [3].

Consider now special case, as in our system, where controllers are simple gains. Control vector is given by 


$$
u(t)=\left[\begin{array}{lll}
C_{m_{k}}\left(x_{e}-x_{m}\right) & C_{s_{k}}\left(x_{m}-x_{e}\right) & f_{h}^{*}
\end{array}\right]^{T}
$$

Using (5) yields for $H k, k=1,2$

$$
H_{k}=\left[\begin{array}{cccc} 
& A_{k} & 0_{4 * 2} & \\
& & \\
\hline C_{m k} & -C_{m k} & 0_{2 * 4} \\
-C_{s k} & C_{s k} &
\end{array}\right]
$$

Close examination to (6) reveals that system's stability is related to matrix $A$ that is the Telerobotic system dynamic. It is easy to show that all A's eigenvalues have negative real part.

\section{CONCLUSION}

In this paper, a new indicator for gain switching controller of a teleoperation system is proposed. Identification loop is just applied to identify gradual changes in slave dynamic parameters. As a result, comparing to Ni's switching controller [1], the time required to estimate abrupt changes of environment in hard contact is omitted. The data required to indicate gain switching instance are explored through environment force observation using an accurate slave dynamic model. Experimental results for both negligible time delay and 150ms time delay in the communication channel are included to show the effectiveness of the proposed controller. Based on stability analysis for the class of gain switching linear systems, stability discussion is presented to ensure the stability of the proposed controller.

\section{REFERENCES}

[1] Liya Ni and W. L. Wang, "A Gain-switching Control Scheme for Position-error-based Force-reflecting Teleoperation,” IEEE $10^{\text {th }}$ Symp. On Haptic Interface For Virtual Envir. \& Teleoperator Sys.,2002

[2] K.Hashtrudi-Zaad and S. E. Salcudean, “Adaptive Transparent Impedance Reflecting Teleoperation,” IEEE Int. Conf. on Robotics and Automation, vol.2, pp.1369-1374, 1996.

[3] Kai Wulff, Fabian Wirth and Robert Shorten, "On the stabilization of a class of SISO switched linear systems," IEEE Int. Conf. on Decision and Control, and the European Control Conference, Seville, Spain, December 12-15, 2005

[4] D. Lawrence, "Stability and Transparency in Bilateral Teleoperation", IEEE Transactions on Robotics and Automation, vol.9, pp.624-637, 1993.

[5] Mc. Cavusoglu, A. Sherman, and F. Tendick, "Design of bilateral teleoperation controllers for haptic exploration and telemanipulation of soft environment” ,IEEE transactions on robotic and automation, August 2002.

[6] B. Hannaford, 'Time-domain passivity control of haptic interfaces," IEEE Transactions on Robotics and Automation, Vol. 18, pp. 1-10, Feb 2002.

[7] K.Hashtrudi-Zaad and S. E. Salcudean, "On the Use of Local Force Feedback for Transparent Teleoperation," IEEE Int. Conf. on Robotics and Automation, May, 1999.

[8] B. Hannaford, "A Design Framework for Teleoperators with Kinesthetic Feedback”, IEEE Trans. of Robotics and Automation, Vol. 5, No. 4, pp. 426-434, 1989.

[9] B. Hannaford and R.J. Anderson, "Experimental and Simulation Studies of Hard Contact in Force Reflecting Teleoperation", Pmc.IEEE Int. Conf.Robot.Automat., 1988, pp.584-589.

[10]Liya Ni and W. L. Wang, "Contact Transition Stability Analysis for a Bilateral Teleoperation System," IEEE Int. Conf. on Robotics \& Automation, Washington, DC, May, 2002.

[11]G. Niemeyer and J. J.Slotine , "Stable Adaptive Teleoperation," IEEE Journal of Oceanic Engineering, vol.16, no.1, pp.152-162, 1991.

[12]Brooks T.L., “Telerobotic Response Requirements,” Proc. IEEE Int. Conf. Sys., Man, \& Cyber., vol. 8, pp. 113\{120, 1990

[13]Kazerooni H., Contact instability of the direct drive robot when constrained by a rigid environment, IEEE Transactions on Automation and Control, Vol. 35 Issue 6, Jun. 1990 pp. 710 -714.

[14]Yokokohji and T. Yoshikawa, "Bilateral control of master-slave manipulators for ideal kinesthetic coupling-formulation and experiment," IEEE Transactions on Robotics and Automation, vol. 10, no. 5, pp. 605-620, October 1994. 